\title{
Title of review article:
}

The role of the multi-parametric MRI in the diagnosis of prostate cancer in biopsy-naïve men.

\section{Author(s):}

Adrian Rhudd ${ }^{123}$, Jean McDonald ${ }^{1}$, Mark Emberton ${ }^{3,4}$, Veeru Kasivisvanathan ${ }^{3,4 \dagger}$

\section{Authors' affiliations:}

${ }^{1}$ University of the West Indies, Mona

${ }^{2}$ Department of Urology, North Middlesex University Hospital

${ }^{3}$ Division of Surgery and Interventional Sciences, University College London

${ }^{4}$ Department of Urology, University College London Hospital

\section{+Corresponding Author:}

Veeru Kasivisvanathan,

Urology Research Group,

Room 4.23, 4th Floor,

132 Hampstead Road,

London,

NW1 2PT

Email: veeru.kasi@ucl.ac.uk

Tel: +44(0)207679 9092, Fax: +44(0)2076799511

\section{Financial Support and Sponsorship:}

Veeru Kasivisvanathan is funded by a Doctoral Research Fellowship from the National Institute for Health Research. Mark Emberton's research is supported by core funding from the United Kingdom's National Institute of Health Research (NIHR) UCLH/UCL Biomedical Research Centre. He was awarded NIHR Senior Investigator in 2015. The views expressed in this publication are those of the 
authors and not necessarily those of the NHS, the National Institute for Health Research or the Department of Health.

\section{Conflicts of Interest:}

The other authors declare no competing interests. 


\begin{abstract}
$\underline{\text { Abstract }}$
Purpose of review: To review the role of pre-biopsy multi-parametric MRI in biopsy-naïve men for the detection of clinically significant prostate cancer.
\end{abstract}

Recent findings: Recent Level 1 evidence shows that multi-parametric MRI has high sensitivity and negative predictive value for the detection of clinically significant prostate cancer in biopsy-naïve men. Concurrent developments include important work in the standardization of MRI-reporting. The low specificity and positive predictive value of MRI means that biopsy is still necessary following MRI. MRI-targeted prostate biopsy has emerged as an alternative diagnostic test to transrectal ultrasound guided prostate biopsy, though its exact role in biopsy naïve men and the optimal technique remain to be defined.

Summary: There is the potential for MRI to be used as a triage test to allow a proportion of men to avoid biopsy and remain on PSA surveillance. MRI-suspicious areas can be sampled more intensively using MRI-targeted biopsy that can be carried out in a variety of ways. Future work should focus on the cost-effectiveness of introducing a pre-biopsy MRI pathway in biopsy naïve men and addressing the training needs for such a change.

\title{
Keywords:
}

MRI, Prostate Cancer, diagnosis, prostate biopsy

\section{Video abstract}




\section{Introduction}

Prostate cancer is the second commonest cancer in men in the world[1]. Prostate specific antigen (PSA) testing forms the basis for screening men at risk of prostate cancer for further investigation. The benefit of PSA screening has been widely debated as there is a fine margin between offering a survival benefit and inflicting harm from overtreatment [2, 3]. The United States Preventive Services Task Force (USPSTF) recommended against prostate cancer screening and gave it a grade D recommendation [4]. There is therefore a need to optimize diagnostic strategies that identify men with clinically significant cancer who will benefit from treatment.

Advances in prostate MRI tend to suggest that it should have a key role in the prostate cancer management algorithm, particularly before a biopsy is done. Recent studies such as the PROMIS Trial raise an important question as to whether prebiopsy multi-parametric MRI should have a routine role in biopsy naïve men for the identification of prostate cancer[5]. 


\section{The role of MRI in the current prostate cancer diagnostic pathway for biopsy naïve men}

Many current practice guidelines, such as the European Association of Urology, the National Institute of Health Care and Excellence (NICE) and the American Urology Association guidelines endorse a diagnostic pathway that includes a digital rectal examination (DRE) and PSA typically followed by a 10-12 core transrectal-ultrasound guided (TRUS) guided prostate biopsy if either of the two is abnormal.[6] [7] [8].

The use of pre-biopsy MRI in the routine prostate cancer guidelines appears to be lagging behind adoption of the technology in many centers. The National Comprehensive Cancer Network (NCCN) in their 2016 guidelines state that "MRI is not recommended routinely prior to initial prostate biopsy" however they do acknowledge that MRI/US fusion targeted biopsy may increase the detection of clinically significant higher risk disease while lowering the detection of lower risk disease[9]. However, in order to recommend MRI routinely both diagnostic accuracy and cost-effectiveness should be clearly demonstrated. The UK NICE guidelines on prostate cancer: diagnosis and management will be updated in mid 2017 and it is likely that guidelines on the use of pre-biopsy MRI in biopsy naïve men will be featured especially in light of the recent publication of the PROMIS Trial $[5,10]$. 


\section{Problems with the existing diagnostic pathway that MRI may potentially solve}

The current screening pathway of DRE, PSA then TRUS biopsy has its limitations. The European Randomized Screen for Prostate (ERSPC) Trial found that $75.9 \%$ of men with a PSA greater than $3.0 \mathrm{ng} / \mathrm{ml}$ that went on to have a TRUS biopsy were negative for cancer. They also found a cumulative risk of $12 \%$ of at least 1 false positive PSA after 3 rounds of PSA testing every 4 years. The Rotterdam Centre had a rate of overdiagnosis as high as $66 \%$ [3].

The Standard systematic 12 core TRUS biopsy has a sensitivity ranging from $45-75 \%$ for clinically significant prostate cancer[11, 12] [13]. Transperineal Template mapping biopsy (TTMB) has sought to improve on these sensitivities by taking more cores and sampling the anterior and midline prostate which can be difficult to reach via the trans rectal route. It also reduces the risks of sepsis associated with the trans rectal route. However TTMB usually requires dedicated equipment, expertise, spinal or general anesthesia and can result in greater overdetection of clinically insignificant cancer [14].

Adverse events associated with the TRUS biopsy procedure are well documented. Hematuria/hematospermia rates are between 20-50\%. Fever post biopsy is between $3.5-4.2 \%$ [3]. More serious complications were found in 68/100,000 biopsies i.e. infections, bleeding and urinary difficulties [2]. Hospitalization post biopsy was found to be $4 \%$ [15]. Of concern was the recent finding that a man with a negative TRUS biopsy had a risk of death from prostate 
cancer of $5.2 \%$, which is greater than the lifetime risk of prostate cancer death for the average man $[16,17]$.

By performing a pre-TRUS biopsy mpMRI it may be possible to:

Reduce the number of biopsies performed, thereby, reducing over diagnosis, false positive rates and adverse events associated with biopsy. It may also be possible to target suspicious lesions more accurately to improve sensitivity and specificity for the detection of clinically significant cancer [18].

\section{Improvements in MRI}

When MRI was first considered for prostate cancer diagnosis it lacked sensitivity and specificity when compared to TRUS biopsy as it was limited to the use of anatomic T2 weighted MRI sequences. The rapid optimization of MRI technology has led to functional imaging using diffusion-weighted, dynamic contrast-enhanced and spectroscopic imaging. Combining these functional techniques with the traditional (T1/T2) techniques (parameters) resulted in a multi parametric MRI (mpMRI) which has led to improved diagnostic performance for the detection of high grade lesions [19].

The European Society of Urogenital Radiology and other expert Uroradiology groups have led attempts at standardizing the reporting of prostate mpMRI [20]. The Prostate Imaging Reporting And Data System (PIRADS) and Likert scores for ascribing risk of clinically significant prostate cancer represent significant steps forward in increasing the applicability of the prostate MRI in non-expert centers [21]. It is clear from the experience of multi-centre clinical trials in Prostate 
MRI such as PROMIS and PRECISION (NCT02380027), that optimization of the conduct of the MRI and reporting experience of the radiologists are key factors in determining the performance of the technology.

The Prostate MR Imaging study (PROMIS) provides level 1 evidence for the contemporary diagnostic performance of mpMRI validated against TTPB in biopsy naïve men [5]. 576 men underwent 1.5T mpMRI with a reference standard of TTPB. Sensitivity was $93 \%$, specificity $41 \%$, positive predictive value $51 \%$ and negative predictive value $89 \%$ for the detection of clinically significant cancer (Gleason score $\geq 4+3$ or a maximum cancer core length $6 \mathrm{~mm}$ or longer). MpMRI compared favourably to TRUS biopsy with regards to sensitivity and negative predictive value, though the specificity and positive predictive value of TRUS biopsy were superior. The implications of these results will be discussed in the appropriate sections below where the potential role of mpMRI in the diagnostic pathway for prostate cancer will be discussed (Table 1).

\section{MpMRI as a triage test}

By doing a pre biopsy mpMRI it may be possible to exclude men at low risk of having clinically significant cancer from going on to having a biopsy. To do this, mpMRI needs to have a high negative predictive value for the detection of clinically significant cancer. The negative predictive value of mpMRI for clinically significant prostate cancer varies widely in the available literature. This may depend on the techniques, interpreters or the definition of clinically significant prostate cancer used. A literature review by Futterer et al has found a range from 63-98\%[22]. 
The Prostate MR Imaging Study (PROMIS) trial is one of the largest prospective level $1 \mathrm{~b}$ evidence trials to add evidence to this debate[5]. Several of the centers involved in PROMIS were not specialized academic centers and MRI was carried out on 1.5T machines rather than $3 \mathrm{~T}$ to show the performance of the test in scanners with coil strengths that are more readily accessible to most centers. The landmark finding was that of a negative predictive value of $89 \%$ for the detection of clinically significant prostate cancer (Gleason score $\geq 4+3$ or a maximum cancer core length $6 \mathrm{~mm}$ or longer.) The study design ensured the conduct and reporting of MRIs was tightly controlled and this standardization will need to be replicated in other non-specialist centers if these results wish to be replicated.

However, according to the secondary definition of clinically significant prostate cancer (Gleason score $\geq 3+4$ or a maximum cancer core length $4 \mathrm{~mm}$ ) the negative predictive value was $72 \%$. For some practitioners, missing $28 \%$ of clinically significant cancer according to this less stringent definition may seem high. One should appreciate, however, that men with negative MRIs are not discharged without follow up but continue on PSA surveillance. Further, the results must be appreciated in the context of what men would otherwise get. If it was a TRUS biopsy, the negative predictive value was still lower than MRI in this study. At the very least, the results of this trial allow an informed discussion with a man who has a negative MRI to consider whether he wishes to avoid a biopsy and embark on PSA surveillance.

Before routinely adapting pre-biopsy MRI in all biopsy naïve men, this practice would need to be proven cost effective. Although cost effectiveness in the 
prior negative biopsy group has been demonstrated, we await robust costeffectiveness analysis in the biopsy naïve group [23].

\section{MpMRI as an additional test to guide MRI-targeted biopsy}

If a suspicious lesion has been identified on an MRI, this can be sampled by a targeted biopsy. Currently the three ways to do so are MRI visual estimation/ cognitive registration, MRI-ultrasound fusion and the In-bore MRI guided biopsy. Cognitive biopsies rely on the operator to review the images and make an effort to deliberately sample the suspicious area using TRUS guidance. MRI-Fusion biopsies use software to co-register the MRI prostate contours and lesion location onto the realtime ultrasound. In bore MRI-targeted biopsies are done within an MRI scanner, using MRI compatible biopsy equipment.

There are few original studies in MRI-targeted biopsies that are appropriately powered to be able to tell if there is a difference between clinically significant cancer detection by MRI-targeted biopsy and TRUS-biopsy. An ongoing prospective randomized trial, PRECISION (NCT02380027), will attempt to address this, comparing CDRs of MRI-targeted prostate biopsy to TRUS biopsy in 470 biopsy naïve men. There are however many smaller studies already published from specialist centers which have found significant improvements in CDRs, particularly with regards to clinically significant cancer, and lower detection rates of clinically insignificant cancer rates using MRI guided techniques [24-29]. A metanalysis by Schoots found a higher detection rate of clinically significant cancer and a lower 
detection rate of clinically insignificant cancer with MRI targeted biopsy techniques. Significant improvement in detection was particularly seen in a subset of men with a prior negative biopsy. [30]. It has not been established whether one particular technique for MRI-targeted biopsy is superior to another. The PROFUS trial, a prospective blinded comparison of the CDRs of cognition TRUS biopsy and MRI/TRUS fusion showed no statistically significant difference in CDRs between the two types of MRI-targeted biopsy registration techniques however there was a trend towards improved CDRs in their subgroup analysis. The authors suggest that these results may be due to the fact that there were only 125 patients in the study[31].

Data from some of the key recent studies in biopsy naïve men evaluating MRI targeted biopsy are summarized in Table 2. Panebianco et al carried out a randomized controlled trial in 1140 men. In Group A, all men underwent TRUS biopsy. In group B, all men underwent pre-biopsy MRI, TRUS biopsy and in addition, MRI-targeted cognitive biopsy in the presence of a suspicious lesion. They found higher CDRs in Group B (73\%) versus group A (38\%)[32]. Though other studies follow a similar trend, the wide differences seen in this well powered study have not been seen in other studies.

A randomized controlled trial in 130 men by Tonttila et al compared a group of men undergoing standard TRUS biopsy to a group of men with a pre-biopsy MRI then standard TRUS + MRI/Fusion biopsies of MRI suspicious lesions where present. Cancer detection was 34/53 (64\%) in the MRI arm and 34/60 (57\%) in the TRUS biopsy arm. Significant cancer detection was 29/53 (55\%) and 27/60 (45\%), 
respectively. There were no statistically significant differences identified, though the comparison was underpowered. [28].

Siddiqui et al reported a prospective paired cohort study in 1003 men undergoing pre-biopsy MRI, TRUS biopsy and MRI-targeted biopsy. 196 men were biopsy naïve and the remaining had a prior negative biopsy. Though there was no difference in overall CDRs between TRUS biopsy and targeted MRI/fusion, 30\% more high-grade cancer was diagnosed with targeted biopsy than TRUS biopsy cores (173 cases versus 122 cases). [27]. This study highlights one of the key advantages of the MRI-influenced pathway - the ability to identify significant cancer whilst avoiding the diagnosis of clinically insignificant cancer.

Another important consideration is whether an MRI-targeted only biopsy approach (i.e. no additional systematic cores) can be taken or whether additional systematic cores need to be taken from MRI-negative areas. Baco et al performed a randomized control trial on 175 biopsy naïve men comparing a standard TRUS biopsy and 2 core TRUS targeted biopsy of DRE or TRUS suspicious lesion versus a 2-core MRI/TRUS fusion biopsy and standard TRUS biopsy. They found no significant difference in overall cancer detection rates ( $54 \%$ vs $59 \%$; p value 0.4 ) or clinically significant prostate cancer detection rates (49\% vs 44\%; p value 0.5 ) between the two groups. Interestingly, they found no statistically significant difference in the clinically significant CDR between a 2 core MRI/TRUS fusion biopsy vs standard biopsy ( $38 \%$ vs $49 \%$; $p$ value 0.2 ). This tends to suggest a targeted 2 core approach may be sufficient[33]. 
Urologists should carefully consider the balance between biopsy core number, clinically significant and clinically insignificant disease detection and not always assume taking more biopsies is the best strategy. We should consider that we are in a resource-limited environment and an MRI-targeted only approach, which would result in fewer biopsies and fewer men diagnosed with insignificant cancer will have health economic and service delivery advantages.

Though equipment for fusion and in-bore MRI techniques may be costly, this may be balanced out by costs saved if fewer men are biopsied and fewer biopsy cores per patient are taken. Issues on training non-specialist centers must also be considered[34].

\section{CONCLUSION}

There have been recent notable developments in mpMRI technology, conduct and reporting to improve the use of MRI in prostate cancer management. These have resulted in clear improvements in the performance of pre-biopsy mpMRI. Level $1 \mathrm{~b}$ evidence is now available demonstrating good performance of mpMRI in biopsy-naïve men in ruling out high grade prostate cancer and support the possibility of its use as a triage test for deciding which men may be able to avoid a biopsy. Some evidence also exists supporting the role of mpMRI-targeted biopsy as an adjunct or replacement test to TRUS-biopsy, though questions remain on training and implementation practicalities. We also await robust cost-effectiveness data to support routine use of a pre-biopsy MRI-influenced diagnostic pathway. What is 
certain is that pre-biopsy mpMRI will play an increasingly important role in biopsy naïve men in years to come. 


\section{Key points}

- Level 1 evidence shows that pre-biopsy multiparametric MRI has a high sensitivity and negative predictive value for the detection of clinically significant prostate cancer in biopsy naïve men.

- Prostate MRI may be able to be used as a triage test to allow a proportion of men to avoid biopsy and remain on PSA surveillance

- The low specificity and positive predictive value of MRI means that prostate biopsy is still necessary. MRI-targeted biopsy appears to be a reasonable alternative to standard 12-core transrectal ultrasound guided prostate biopsy though the optimal technique and its exact role in biopsy naïve men remains to be established

- Analyses showing cost-effectiveness of an MRI-driven diagnostic pathway in biopsy naïve men are awaited 


\section{$\underline{\text { Tables }}$}

Table 1: The potential roles for mpMRI in diagnosing prostate cancer in biopsynaïve men (original table):

1. After Digital rectal examination and Prostate specific antigen testing as a triage test to decide on which men can avoid biopsy and which need to go onto biopsy.

2. After Digital rectal examination and Prostate specific antigen testing as an additional test to guide MRI-targeted biopsy, whether cognition, fusion or inbore MRI guided. 


\begin{tabular}{|c|c|c|c|c|c|c|c|}
\hline Study (year) & Study Design & Intervention(s) & Sample size & Population & $\begin{array}{l}\text { Overall cancer } \\
\text { detection }\end{array}$ & $\begin{array}{l}\text { Significant cancer } \\
\text { detection }\end{array}$ & Conclusions \\
\hline $\begin{array}{l}\text { Panebianco } \\
\text { 2015[32] }\end{array}$ & RCT & $\begin{array}{l}\text { Group A: SB } \\
\text { Group B: MRI+ MRI- } \\
\text { targeted \& SB }\end{array}$ & 1140 & Biopsy naive & $\begin{array}{l}\text { Group A: } 215 / 570 \\
(37 \%) \\
\text { Group B: } 417 / 570 \\
(73 \%)\end{array}$ & Not specified & $\begin{array}{l}\text { The CDRs of prostate cancer is } \\
\text { higher among those randomized } \\
\text { toMRI then SB with targeted } \\
\text { cores vs SB alone }\end{array}$ \\
\hline Tonttila 2016[28] & RCT & $\begin{array}{l}\text { Control: SB } \\
\text { Intervention: } \\
\text { MRI+SB+fusion }\end{array}$ & 130 & Biopsy naive & $\begin{array}{l}\text { Control: } 34 / 60(57 \%) \\
\text { Intervention: } 34 / 53 \\
(64 \%)\end{array}$ & $\begin{array}{l}\text { Control: } 27 / 60 \\
(45 \%) \\
\text { Intervention: } \\
29 / 53(55 \%) \\
\end{array}$ & $\begin{array}{l}\text { Fusion did not improve CDRs } \\
\text { when compared to SB alone }\end{array}$ \\
\hline Pokorny 2014[24] & $\begin{array}{l}\text { Prospective } \\
\text { cohort study }\end{array}$ & $\begin{array}{l}\text { Group A: MRI + SB } \\
\text { Group B: MRI + SB } \\
\text { +MRCB }\end{array}$ & 223 & Biopsy naive & $\begin{array}{l}\text { SB:126/223 (67\%) } \\
\text { MRCB: } 99 / 142(70 \%)\end{array}$ & $\begin{array}{l}\text { SB: 79/126 (63\%) } \\
\text { Group B:Int/high } \\
\text { risk 93/99 (94\%) }\end{array}$ & $\begin{array}{l}\text { MRI/MRGB reduces the } \\
\text { detection of low risk cancer and } \\
\text { improves intermediate and high } \\
\text { risk cancer detection rate }\end{array}$ \\
\hline Siddiqui 2015[27] & $\begin{array}{l}\text { Prospective } \\
\text { cohort study }\end{array}$ & MRI+ fusion + SB & $\begin{array}{l}1003 \\
\text { (196 Biopsy } \\
\text { naïve) }\end{array}$ & Mixed & $\begin{array}{l}\text { Fusion: 461/1003 } \\
(46 \%) \\
\text { SB: 469/1003 (47\%) }\end{array}$ & $\begin{array}{l}\text { Fusion: } 173 / 461 \\
(38 \%) \\
\text { SB: } 122 / 469 \text { (26\%) }\end{array}$ & $\begin{array}{l}\text { Fusion biopsy associated with } \\
\text { increased CDRs of high risk } \\
\text { prostate cancer and decreased } \\
\text { detection of low risk prostate } \\
\text { cancer but no difference in } \\
\text { overall CDRs }\end{array}$ \\
\hline $\begin{array}{l}\text { Porpiglia } \\
\text { 2016[29] }\end{array}$ & RCT & $\begin{array}{l}\text { Group A: MRI + SB or } \\
\text { fusion } \\
\text { Group B: SB }\end{array}$ & 212 & Biopsy naive & $\begin{array}{l}\text { Group A: } 54 / 107 \\
(50.5 \%) \\
\text { Group B: } 31 / 105 \\
(29.5 \%)\end{array}$ & Not specified & $\begin{array}{l}\text { Pathway which incorporates MRI } \\
\text { has a higher CDR for prostate } \\
\text { cancer when compared to the } \\
\text { pathway without MRI }\end{array}$ \\
\hline Baco 2016[33] & RCT & $\begin{array}{l}\text { Control: SB+ } \\
\text { DRE/TRUS targeted } \\
\text { suspicious lesions } \\
\text { Intervention: SB+ } 2 \\
\text { core fusion }\end{array}$ & 175 & Biopsy Naive & $\begin{array}{l}\text { Control: } 48 / 89(54 \%) \\
\text { Intervention: } 51 / 86 \\
(59 \%)\end{array}$ & $\begin{array}{l}\text { Control: } 44 / 89 \\
(49 \%) \\
\text { Intervention: } \\
38 / 86(44 \%)\end{array}$ & $\begin{array}{l}\text { No difference was found in } \\
\text { overall CDRs or clinically } \\
\text { significant prostate CDRs } \\
\text { between SB and fusion. A 2-core } \\
\text { targetd fusion biopsy approach } \\
\text { was equivalent to SB }\end{array}$ \\
\hline
\end{tabular}

Legend: (SB: Standard TRUS Biopsy. Fusion: MRI/TRUS fusion. MRCB: MRI-targeted cognitive biopsy. CDRs: Cancer detection rates)

Table 2: A summary of the key recent studies evaluating MRI-targeted prostate biopsy in biopsy naïve men (original table) 


\section{References}

1. Ferlay, J., et al., Cancer incidence and mortality worldwide: sources, methods and major patterns in GLOBOCAN 2012. Int J Cancer, 2015. 136(5): p. E35986.

2. Andriole, G.L., et al., Prostate cancer screening in the randomized Prostate, Lung, Colorectal, and Ovarian Cancer Screening Trial: mortality results after 13 years of follow-up. J Natl Cancer Inst, 2012. 104(2): p. 125-32.

3. Schroder, F.H., et al., Screening and prostate-cancer mortality in a randomized European study. N Engl J Med, 2009. 360(13): p. 1320-8.

4. $\quad$ Moyer, V.A. and U.S.P.S.T. Force, Screening for prostate cancer: U.S. Preventive Services Task Force recommendation statement. Ann Intern Med, 2012. 157(2): p. 120-34.

**5. Ahmed, H.U., et al., Diagnostic accuracy of multi-parametric MRI and TRUS biopsy in prostate cancer (PROMIS): a paired validating confirmatory study. Lancet, 2017. This is the largest randomised controlled multicentr trial to provide level $1 \mathrm{~b}$ evidence of the seuperior negative predictive value of clinically significant prostate cancer in biopsy naiive men.

6. Mottet, N., et al., EAU-ESTRO-SIOG Guidelines on Prostate Cancer. Part 1: Screening, Diagnosis, and Local Treatment with Curative Intent. Eur Urol, 2016.

7. Graham, J., et al., Prostate cancer: summary of updated NICE guidance. BMJ, 2014. 348: p. f7524.

8. Carter, H.B., et al., Early detection of prostate cancer: AUA Guideline. J Urol, 2013. 190(2): p. 419-26.

9. Carroll, P.R., et al., NCCN Guidelines Insights: Prostate Cancer Early Detection, Version 2.2016. J Natl Compr Canc Netw, 2016. 14(5): p. 509-19.

10. Excellence, N.I.f.H.a.C. Surveillance report 2016 - Prostate cancer: diagnosis and management (2014) NICE guidelines CG175. 2016.

11. Haas, G.P., et al., Needle biopsies on autopsy prostates: sensitivity of cancer detection based on true prevalence. J Natl Cancer Inst, 2007. 99(19): p. 14849.

12. Rocco, B., et al., Sensitivity and detection rate of a 12-core trans-perineal prostate biopsy: preliminary report. Eur Urol, 2006. 49(5): p. 827-33.

13. Jones, J.S., Saturation biopsy for detecting and characterizing prostate cancer. BJU Int, 2007. 99(6): p. 1340-4.

14. Ting, F., et al., Assessment of the Performance of Magnetic Resonance Imaging/Ultrasound Fusion Guided Prostate Biopsy against a Combined Targeted Plus Systematic Biopsy Approach Using 24-Core Transperineal Template Saturation Mapping Prostate Biopsy. Prostate Cancer, 2016. 2016: p. 3794738.

15. Loeb, S., et al., Complications after prostate biopsy: data from SEER-Medicare. J Urol, 2011. 186(5): p. 1830-4.

16. Klemann, N., et al., Risk of prostate cancer diagnosis and mortality in men with a benign initial transrectal ultrasound-guided biopsy set: a population-based study. Lancet Oncol, 2017. 18(2): p. 221-229. 
17. Emberton, M., Is a negative prostate biopsy a risk factor for a prostate cancer related death? Lancet Oncol, 2017. 18(2): p. 162-163.

18. Ridout, A.J., et al., Role of magnetic resonance imaging in defining a biopsy strategy for detection of prostate cancer. Int J Urol, 2014. 21(1): p. 5-11.

19. Thompson, J., et al., The role of magnetic resonance imaging in the diagnosis and management of prostate cancer. BJU Int, 2013. 112 Suppl 2: p. 6-20.

20. Dickinson, L., et al., Magnetic resonance imaging for the detection, localisation, and characterisation of prostate cancer: recommendations from a European consensus meeting. Eur Urol, 2011. 59(4): p. 477-94.

21. Vargas, H.A., et al., Updated prostate imaging reporting and data system (PIRADS v2) recommendations for the detection of clinically significant prostate cancer using multiparametric MRI: critical evaluation using wholemount pathology as standard of reference. Eur Radiol, 2016. 26(6): p. 160612.

*22. Futterer, J.J., et al., Can Clinically Significant Prostate Cancer Be Detected with Multiparametric Magnetic Resonance Imaging? A Systematic Review of the Literature. Eur Urol, 2015. 68(6): p. 1045-53. A 14 year literature rivew of the negative predictive value of mpMRI for prostate cancer detection ranges from $63-98 \%$.

23. Mowatt, G., et al., The diagnostic accuracy and cost-effectiveness of magnetic resonance spectroscopy and enhanced magnetic resonance imaging techniques in aiding the localisation of prostate abnormalities for biopsy: a systematic review and economic evaluation. Health Technol Assess, 2013. 17(20): p. viixix, 1-281.

24. Pokorny, M.R., et al., Prospective study of diagnostic accuracy comparing prostate cancer detection by transrectal ultrasound-guided biopsy versus magnetic resonance (MR) imaging with subsequent MR-guided biopsy in men without previous prostate biopsies. Eur Urol, 2014. 66(1): p. 22-9.

25. Mendhiratta, N., et al., Magnetic Resonance Imaging-Ultrasound Fusion Targeted Prostate Biopsy in a Consecutive Cohort of Men with No Previous Biopsy: Reduction of Over Detection through Improved Risk Stratification. J Urol, 2015. 194(6): p. 1601-6.

26. Meng, X., et al., Relationship Between Prebiopsy Multiparametric Magnetic Resonance Imaging (MRI), Biopsy Indication, and MRI-ultrasound Fusiontargeted Prostate Biopsy Outcomes. Eur Urol, 2016. 69(3): p. 512-7.

*27. Siddiqui, M.M., et al., Comparison of MR/ultrasound fusion-guided biopsy with ultrasound-guided biopsy for the diagnosis of prostate cancer. JAMA, 2015. 313(4): p. 390-7. This is a large prospective cohort study showing no difference in overall cancer detection rate but significantly better cancer detection rates for clinically significant prostate cancer. This compared standard TRUS biopsy to MRI/TRUS fusion biopsy.

*28. Tonttila, P.P., et al., Prebiopsy Multiparametric Magnetic Resonance Imaging for Prostate Cancer Diagnosis in Biopsy-naive Men with Suspected Prostate Cancer Based on Elevated Prostate-specific Antigen Values: Results from a Randomized Prospective Blinded Controlled Trial. Eur Urol, 2016. 69(3): p. 
419-25. A randomised control trial showing no difference in CDRs between TRUS biopsy and MRI/TRUS fusion biopsy techniques

29. Porpiglia, F., et al., Diagnostic Pathway with Multiparametric Magnetic Resonance Imaging Versus Standard Pathway: Results from a Randomized Prospective Study in Biopsy-naive Patients with Suspected Prostate Cancer. Eur Urol, 2016.

**30. Schoots, I.G., et al., Magnetic resonance imaging-targeted biopsy may enhance the diagnostic accuracy of significant prostate cancer detection compared to standard transrectal ultrasound-guided biopsy: a systematic review and metaanalysis. Eur Urol, 2015. 68(3): p. 438-50. This is the first metanalysis comparing TRUS to MR guided targeted biopsies showing significant improvement in cancer detection rates with MR targeted biopsy techniques.

31. Wysock, J.S., et al., A prospective, blinded comparison of magnetic resonance (MR) imaging-ultrasound fusion and visual estimation in the performance of MR-targeted prostate biopsy: the PROFUS trial. Eur Urol, 2014. 66(2): p. 34351.

*32. Panebianco, V., et al., Multiparametric magnetic resonance imaging vs. standard care in men being evaluated for prostate cancer: a randomized study. Urol Oncol, 2015. 33(1): p. 17 e1-7. This is one of the largest randomised control trials showing superior overall cancer detection rates for an MRI guided based pathway compared to standard TRUS.

33. Baco, E., et al., A Randomized Controlled Trial To Assess and Compare the Outcomes of Two-core Prostate Biopsy Guided by Fused Magnetic Resonance and Transrectal Ultrasound Images and Traditional 12-core Systematic Biopsy. Eur Urol, 2016. 69(1): p. 149-56.

34. Venderink, W., et al., Cost-Effectiveness Comparison of Imaging-Guided Prostate Biopsy Techniques: Systematic Transrectal Ultrasound, Direct In-Bore MRI, and Image Fusion. AJR Am J Roentgenol, 2017: p. 1-6. 